\title{
Clinical Challenges in Using Chinese Medicinal Herbs
}

\author{
Tong Zheng* \\ Institute of Healthcare, Taiwan
}

*Corresponding author: Tong Zheng, Institute of Healthcare, Taiwan

Received Date: July 01, 2019

Published Date: July 18, 2019

\begin{abstract}
Clinical use of Chinese medicinal herbs face challenges, such as medicinal herbs or granules, dosage, accurate selections of herbs, to use unprocessed or not to use processed medicinal herbs, and cooking time. Pattern identification, which has seldom been taken into consideration in current research examining the safety and efficacy of Chinese medicinal herbs, plays a key to understanding the successful role of clinical use of Chinese medicinal herbs.
\end{abstract}

Keywords: Medicinal herb; Herbal ingredients; processed; Pattern identification

\section{Introduction}

After acupuncture that has gained popularity and is recognized as the part of the healthcare system in the West, Chinese herbs are also getting more and more attention with the news that Chinese herbs are accepted and used by Olympic athletes, even though they are still viewed as the complementary and alternative healthcare tools. Traditional Chinese medicine (TCM) is developed on the basis of inductive logic thinking that focuses on the similarity through the observation of nature. Under this notion, Chinese herbs develops its own theories different from those of acupuncture, which to some extents can be complicated to understand for clinical use and learning. A pharmaceutical consideration is required in clinical use of Chinese herbs when the desired outcomes are expected for an individual's conditions. Processing induces expected actions that can vary depending on the factors, such as timing, processing approaches, etc.

\section{Current Challenges}

Traditional Chinese medicine (TCM) preparations in the treatments are often prescribed according to an individuals' pattern identification, which has the pathological changes of diseases based on the TCM theories derived from Yin-Yang, Five Elements, and QiBlood. In addition to the accurate pattern identification, whether or not a Chinese herb in a preparation can be effective for expected outcomes, issues like accurate selections, the use of processed/ unprocessed, and the considerations of herbal characteristics are the keys.

\section{Medicinal herbs or granules}

It's been used for more than 20 years with dispensing granule convenient to patients; however, it remains uncertain and deserves attention whether or not Chinese herbal medicine granules can work more effectively than combinations of medicinal herbs, in addition to safety, which affects greatly TCM practitioners' clinical considerations. The current clinical controlled trials (CCTs), including randomized trials (RCTs), show the comparisons of the effectiveness and safety between Chinese medicinal herbs and herbal medicine granules cannot reach a definitive conclusion in clinical practice [1].

\section{Dosage}

The essential part of a Chinese herbal medicinal formula lies in the dose of herbal ingredients. The adjustment of the ratio of herbal ingredients in the formula is generally determined by TCM practitioners, analyzing the improvement or aggravation of the symptoms to achieve an expected therapeutic effect. Different ratios of herb ingredients in a formula may totally alter the therapeutic effects and generally result in different clinical outcomes. The result shows a greater proportion of Salvia miltiorrhiza Bge. used in the Danshen-Sanqi formula for cardiovascular management, the better 
outcome is expected. However, when the formula is used to treat haemostasis, the dosage of Danshen should be reduced [2].

\section{Accurate selections of herbs}

In terms of treating Liver-related diseases, it is beyond doubt that Chai Hu (Bupleurum chinense DC. and Bupleurum scorzonerifolium Willd) in TCM is the well-known herb composed in Xiao-yao-san (逍遙散) and Xiao Chai Hu decoction (小柴胡 湯), the most frequently used formulas for more than 2000 years. Chaihu bearing the properties of spicy, cool, and bitter was initially recorded in $200 \mathrm{AD}$ in the well-known TCM classic Shennongbencao-jing (神農本草經) and used for dispersing the evil heat to sooth stagnated Liver qi to treat the antiviral, anti-inflammatory, antimicrobial, and immunomodulatory issues.

However, whether or not Bei Chai Hu (北柴胡, Bupleurum chinense DC.) should be only selected as the components of these two formulas remains controversial because it has not been specified by the author Zhang Zhongjing of Shang Han Lun (傷寒 論), even though Bupleurum chinense DC. is mostly selected for clinical use.

Clinical use traditionally in the Chinese communities, Chai $\mathrm{Hu}$ refers to the dried root of either Bupleurum chinense DC. or Bupleurum scorzonerifolium Willd (南柴胡, Nan Chai Hu) selected for the clinical needs. The major differences between Bupleurum chinense DC. and Bupleurum scorzonerifolium Willd are that the indication of Bupleurum chinense DC. is dispersing stagnated Liver qi while Bupleurum scorzonerifolium Willd is raising Yang Qi in the pattern of Middle Jiao deficiency [3].

For clinical use with indications changed to meet the patients' needs, crude, fried, or vinegar-baked Radix Bupleuri may be usually selected. To use unprocessed or not to use processed medicinal herbs in ancient times before humans began the use of fire, Chinese herbs were only cleaned with water for use to ills. With the changes of time, methods of processing are developed and conducted in stirfrying, steaming, boiling, water trituration, calcining, etc.

TCM sees the body as a whole system and a miniature of the universe. Patients are diagnosed strictly following the YinYang theory and the Five Elements theory, which serves as the guidance for prescriptions [4]. TCM and acupuncture, emphasize an individual's pattern that is totally unique and different from the symptoms from the aspect of the Western medicine. In other words, TCM is the customer-made medicine that takes specific considerations of the prescriptions consisting of raw or processed herbs to the patients.

Bupleuri Radix can be baked with vinegar for specific purposes. It is reported that vinegar baked Bupleuri Radix can be more effective for relieving the depressed liver and bring about the better antidepressant effect than unprocessed Bupleuri Radix. In addition, the levels of neurotransmitters in the frontal cortex and hippocampus can be regulated significantly through the use of vinegar baked Bupleuri Radix [5].

\section{Cooking time}

In terms of efficacy of medicinal herbs, heed should be paid to cooking time of Chinese herbal medicine. In addition to the hepatoprotective and the chemo preventive effects and the capability to improve the antioxidant defense systems, crude Bupleuri Radix selected in a research was boiled for 4 hours and demonstrated cytotoxicity in HepG2 hepatoma cells induced by 5fluorouracil was enhanced and could protect normal lymphocytes from the cytotoxicity induced by 5 -fluorouracil [6].

\section{Discussion}

Safety and efficacy are the concerns that attract TCM practitioners and patients. How long it should take for cooking if vinegar baked Bupleuri Radix is selected for the expected effects in treating liver diseases highlights the cooking time plays a key role in the efficacy. In other words, whether or not 4 hours to cook crude Bupleuri Radix for the expected results can be the same when crude Bupleuri Radix is only cooked for 3 hours or less remains uncertain.

Dispensing granules are convenient to patients to save time and carry. However, whether Chinese herbal medicine granules and medicinal herbs can have the same degree of effectiveness and safety in clinical practice seems to be impossible to reach the definitive conclusion because there are factors like origin of products, harvest time, processed/unprocessed, etc. On the other hand, there are not sufficient standardizations regulating the ingredients of the granules, including toxicological level. In other words, there are existing disputes if granules can substitute traditional decoctions since there is not enough high quality evidence of efficacy and safety provided at present.

In the meantime, there is no standardized ratio for a formula in the TCM clinics. The ratio is in general determined and designed by a practitioner based on personal experience or traditional classics. It is difficult to systematically understand and analyze the ratios used in the formulae with the present literature.

In addition to the challenges discussed above, pattern differentiation is required in the acupuncture and the TCM treatments for the expected outcomes because pattern is the unique concept not only different from the Western medicine but also plays the key role. Without the accurate pattern identification, the expected outcomes cannot be gained even though these challenges are taken into consideration. Patterns in the TCM and acupuncture encompass broader symptoms than the Western medicine [7].

\section{Conclusion}

Most of the current research exploring the mechanism of efficacy of Chinese medicine fail to provide the answers to the issues listed. The present literature, to some extents, reveals that most of the research focuses only on disease rather than pattern without considering TCM patterns. There are significant challenges in regulating the safety of Chinese herbs and the understanding of efficacy for practice, which can ensure a similar safety standard and the verification of efficacy as Western medications to convince practitioners and patients from the evidenced-based approach. 


\section{Acknowledgement}

None.

\section{Conflicts of Interest}

No conflicts of interest.

\section{References}

1. Qiu R, Zhang X, Zhao C, Li M, Shang H (2018) Comparison of the efficacy of dispensing granules with traditional decoction: a systematic review and meta-analysis. Ann Transl Med 6(3): 38.

2. Zheng, Q, Peng C, Shen M, Yang M (2009) Study on compatibility of Radix et Rhizoma Salviae miltiorrhizae and Radix et Rhizoma notoginseng. Chin J Exp Trad Med Formulae 15: 83-86.
3. Hong TZ (2019) Suggestions for Clinical Use of Chinese Herbs. Advancements Bioequiv Availab. 2(3).

4. Hong TZ (2018) A Review for Integrating Western and Chinese Medicines in Primary Dysmenorrhea. Advancements Bioequiv Availab 2(1).

5. Lei T, Chen S, Wang K, Zhang D, Dong L, et al. (2017) Characterization and discrimination of raw and vinegar-baked Bupleuri Radix based on UHPLC-Q-TOF-MS coupled with multivariate statistical analysis. Biomedical Chromatography 32(2).

6. Su JK, Lee YJ, Kim BM, Kim Y, Woo H, et al. (2008) Effect of Bupleuri Radix Extracts on the Toxicity of 5Fluorouracil in HepG2 Hepatoma Cells and Normal Human Lymphocytes. Basic \& Clinical Pharmacology \& Toxicology - Basic Clin Pharmacol Toxicol 103(4): 305-313

7. Hong TZ. Stagnated Liver-Qi-Based Insights into the Effects of Vinegarbaked Radix Bupleuri. Advancements Bioequiv Availab 2(4). 